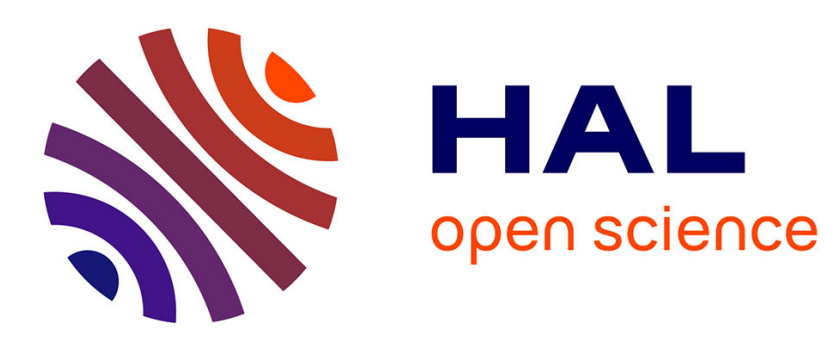

\title{
Asymmetry of vertical buoyancy gradient in stratified turbulence
}

\author{
Andrea Maffioli
}

\section{To cite this version:}

Andrea Maffioli. Asymmetry of vertical buoyancy gradient in stratified turbulence. Journal of Fluid Mechanics, 2019, 870, pp.266-289. 10.1017/jfm.2019.240 . hal-02426830

\section{HAL Id: hal-02426830 https://hal.science/hal-02426830}

Submitted on 2 Jan 2020

HAL is a multi-disciplinary open access archive for the deposit and dissemination of scientific research documents, whether they are published or not. The documents may come from teaching and research institutions in France or abroad, or from public or private research centers.
L'archive ouverte pluridisciplinaire HAL, est destinée au dépôt et à la diffusion de documents scientifiques de niveau recherche, publiés ou non, émanant des établissements d'enseignement et de recherche français ou étrangers, des laboratoires publics ou privés. 


\title{
Asymmetry of vertical buoyancy gradient in stratified turbulence
}

\author{
Andrea Maffioli \\ LMFA, École Centrale de Lyon, 69134 Écully, France
}

(Received xx; revised $\mathrm{xx}$; accepted $\mathrm{xx}$ )

We consider the asymmetry of the buoyancy field in the vertical direction in stratified turbulence. While this asymmetry is known, its causes are not well understood and it has not been systematically quantified previously. Using theoretical arguments, it is shown that both stratified turbulence and isotropic turbulence in the presence of a mean scalar gradient will become positively skewed, as a direct consequence of the presence of stratification and mean scalar gradient, respectively. Assuming a rapid adjustment of isotropic turbulence to a stable stratification on a timescale $\tau \sim N^{-1}$, where $N$ is the Brunt-Väisälä frequency, a scaling for the skewness of the vertical buoyancy gradient is obtained. Direct numerical simulations (DNS) of stratified turbulence with forcing are performed and the positive skewness of $\partial b / \partial z$ is confirmed ( $b$ is the buoyancy). Both the volume-averaged dimensional skewness, $\left\langle(\partial b / \partial z)^{3}\right\rangle$, and the non-dimensional skewness, $S$, are computed and compared against the theoretical predictions. There is a good agreement for $\left\langle(\partial b / \partial z)^{3}\right\rangle$, while there is a discrepancy in the behaviour of $S$. The theory predicts $S \sim 1$ and a constant skewness, while the DNS confirm that the skewness is $O(1)$ but with a remaining dependence on the Froude number. The results are interpreted as being due to the concurrent action of linear and non-linear processes in stratified turbulence leading to $S>0$ and to the formation of layers and interfaces in vertical profiles of buoyancy.

Key words: Authors should not enter keywords on the manuscript, as these must be chosen by the author during the online submission process and will then be added during the typesetting process (see http://journals.cambridge.org/data/relatedlink/jfmkeywords.pdf for the full list)

\section{Introduction}

Stratified turbulence is turbulence in the presence of a stable density stratification. Depending on the strength of the stratification, this turbulent flow can be altered to different degrees by the buoyancy forces that the internal density differences of the fluid bring about. As a result, stratified turbulence can be very different from homogeneous isotropic turbulence. Because of its significance for atmospheric and oceanic turbulence, there has been a continuous fluorishing of research dealing with this subject. In their recent review of mixing in the ocean, Gregg et al. (2018) point out that in the stratified turbulence literature a layer-interface model is often assumed. In this model, the turbulence is seen to evolve and to create regions of partially mixed fluid, with weaker density gradient than the background density gradient (the layers), separated by thin regions of high density gradient (the interfaces). We will refer to this model density profile as a staircase profile, as it is sometimes called in the literature. Note also that 

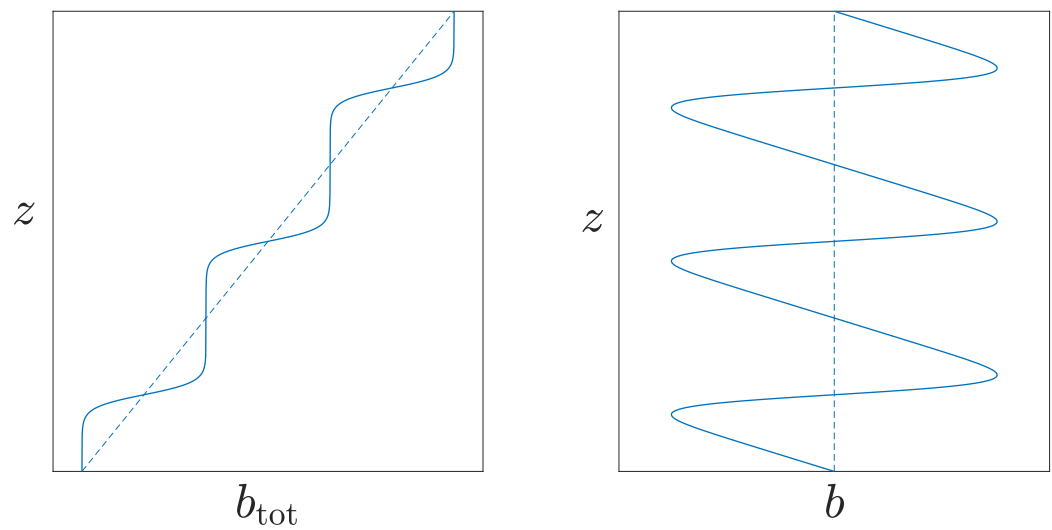

Figure 1. Sketch of the staircase profile of the total buoyancy $b_{\text {tot }}=\bar{b}+b$ and of the corresponding buoyancy profile. In the plot of total buoyancy the dashed line represents the background linear buoyancy profile, $\bar{b}(z)=N^{2} z$.

the high-gradient interfaces are sometimes referred to as sheets and the layers as steps in previous studies (see, e.g., Browand et al. 1987; Gregg et al. 2018).

To describe the layer-interface model quantitatively it is necessary to introduce the total density of the fluid, $\rho=\rho_{0}+\bar{\rho}(z)+\rho^{\prime}(\mathbf{x})$. The three terms making up $\rho$ are a constant reference density $\rho_{0}$, the linear density profile $\bar{\rho}(z)$ and the three-dimensional perturbation $\rho^{\prime}(\mathbf{x})$ created by the turbulent flow. It is worth introducing the buoyancy $b$, a quantity related to the density perturbation in the following way, $b=-\rho^{\prime} g / \rho_{0}$. The buoyancy $b$ is again a perturbation quantity, away from the background buoyancy $\bar{b}$, where $d \bar{b} / d z=N^{2}$. As a result $\langle b\rangle=0$ as well as $\langle\partial b / \partial z\rangle=0$, where $\langle\ldots\rangle$ denotes an average. Note that in terms of the buoyancy the background gradient is positive, in contrast to the background density gradient, which is negative and a more customary quantity. The staircase profile composed of repeating layers and interfaces is sketched in terms of buoyancy in figure 1. Let us imagine for a moment that this profile is a vertical profile of buoyancy measured in a stratified flow. Then it is relatively clear that most of the fluid particles are within layers and so have a total buoyancy gradient that is weaker than the background. On the other hand, a small number of fluid particles have strongly positive total buoyancy gradients, corresponding to the highly stable interfaces in the staircase profile. This feature can also be seen from the plot of the buoyancy $b$, corresponding to the idealized staircase profile, also shown in figure 1 . Now, the profile of $b$ is clearly asymmetric, with large extents of mildly negative $\partial b / \partial z$ and small extents of strongly positive $\partial b / \partial z$. This means that if we consider statistics of the local buoyancy gradient we will obtain results that are not symmetric about $\partial b / \partial z=0$ but that are skewed towards positive values of $\partial b / \partial z$. Hence, this simple and widely used staircase profile contains a statistical asymmetry of the vertical buoyancy gradient. It is here argued that this is an important feature of this turbulent flow which should be considered in more detail than has been done previously.

Such asymmetry in stratified turbulence has first been noted and connected to the staircase profile by Kimura et al. (2016). They performed Direct Numerical Simulations (DNS) of stratified turbulence with forcing, which was vertically coherent and excited only the vortex mode of the stratified flow and not the wave mode (see Riley \& Lelong 2000). The visualisations of buoyancy in a vertical slice showed the presence of layers, and total buoyancy profiles beared some resemblence with the staircase profile idealization of figure 1, while being significantly more irregular. Probability density functions (PDFs) of 
the buoyancy gradients from the DNS had an asymmetric shape for $\partial b / \partial z$ with a positive skewness, while the PDFs of the horizontal buoyancy gradients, $\partial b / \partial x$ and $\partial b / \partial y$, were symmetric. In this study (Kimura et al. 2016), the asymmetry of the vertical buoyancy gradient was not quantified and values of the skewness of $\partial b / \partial z$ were not reported. The skewness of $\partial b / \partial z$ is defined as follows:

$$
S \equiv \frac{\left\langle\left(\frac{\partial b}{\partial z}\right)^{3}\right\rangle}{\left\langle\left(\frac{\partial b}{\partial z}\right)^{2}\right\rangle^{3 / 2}}
$$

In the context of ocean turbulence, Desaubies \& Gregg (1981) reported statistics of vertical temperature gradient in the pycnocline varying the differencing interval or scale; these statistics have a high skewness at relatively fine scales of $\sim 1 \mathrm{~m}$, while the skewness was considerably reduced at larger scales of $\sim 10 \mathrm{~m}$. Similar results on the vertical gradients of vertical isopycnal displacements, which are related to vertical density gradients, were obtained by Pinkel et al. (1991). Both of these studies (Desaubies \& Gregg 1981; Pinkel et al. 1991) found some evidence of layer-interface structure but, as stated by Gregg et al. (2018), "the typical pycnocline is 'irregularly steppy' precluding objective identification of high-gradient sheets separating homogeneous layers". Internal waves were found to be the dominant process at large scales $(\sim 10 \mathrm{~m})$ whereas at the smaller highly-skewed scales $(\sim 1 \mathrm{~m})$ turbulence and mixing events were interpreted as being the important process.

The layer-interface model is relevant also for other turbulent flows. In particular, similar structure is presented by turbulence with a passive scalar in which the scalar fluctuations are created by a mean scalar gradient (Warhaft 2000). If the scalar perturbation $\phi$ is advected by a turbulent flow with a mean scalar gradient in the $z$-direction, $d \bar{\phi} / d z$, then layers and interfaces emerge in $z$-profiles of total scalar concentration $\bar{\phi}+\phi$ (Warhaft 2000). In the literature of passive scalar advection this structure is known as a "rampcliff structure". By the above arguments, this structure results in a non-zero skewness of $\partial \phi / \partial z$, as has long been known in the passive scalar literature (see the review of Warhaft 2000, and references therein). A skewness of $\partial \phi / \partial z$ can be defined analogously to the definition of $S$ in equation 1.1 and this quantity was found to be $\approx 1$ in the grid turbulence experiments with a wall-normal temperature gradient of Mydlarski \& Warhaft (1998). In a recent study, de Bruyn Kops (2015) compared the behaviour of passive scalar advection with a mean scalar gradient to that of stratified turbulence, both of which were simulated using DNS. It was found that in both situations the vertical scalar gradient (corresponding to the vertical buoyancy gradient for the stratified turbulence cases) is positively skewed, and the values reported for the cases with strong stratification are $S \approx 2-2.3$, higher than the value for the experiments with a passive scalar. It therefore appears that a similar structure of the scalar emerges in the presence of a scalar gradient, either in the case the scalar is buoyancy (and so has an effect on the momentum equation) or a passive scalar. In both cases, the scalar is positively skewed but the value of $S$ seems to be higher for strongly stratified turbulence than for passive scalar turbulence.

The physical mechanism or instability causing the layer-interface structure of stratified turbulence is not well understood and is the subject of active debate (Park et al. 1994; Holford \& Linden 1999; Thorpe 2016; Taylor \& Zhou 2017). An instability leading to such structure was proposed early on by Phillips (1972) and Posmentier (1977) and is known as the Phillips-Posmentier instability. In his work, Phillips (1972) considered the 
horizontally-averaged momentum and buoyancy equations in the presence of a mean shear and stratification. The mean (i.e. horizontally-averaged) profiles of horizontal velocity, $\bar{u}$, and buoyancy, $\bar{b}$, of the turbulent flow are then perturbed and an eddy viscosity and diffusivity are used to close the resulting equations for these "mean perturbations". Finally, Phillips found that if the eddy diffusivity decreases sufficiently rapidly with increasing $R i_{g}=N^{2} /(d \bar{u} / d z)^{2}$, then the perturbation of the mean buoyancy profile will grow leading to a layer-interface structure. Here $R i_{g}$ is the gradient Richardson number. Laboratory experiments of homogeneous stratified turbulence in which layering was observed were reviewed by Thorpe (2016) and in a number of cases the above condition on the decrease of the eddy diffusivity with $R i_{g}$ was not satisfied. This condition was satisfied in the experiments only when the turbulence was already layered. This led Thorpe (2016) to conclude that the Phillips-Posmentier instability is not responsible for the initial formation of the layers in these experiments but may reinforce an existing layer-interface structure. An additional mechanism that may create a layer-interface structure is turbulent mixing of a localized "blob" of fluid, which subsequently collapses and spreads laterally as an intrusion due to the horizontal density differences with the ambient stratified fluid. Such an intrusion effectively creates a horizontal layer of relatively well-mixed fluid while spreading; this mechanism was proposed by a number of authors (Browand et al. 1987; Maffioli et al. 2014; Thorpe 2016). Further instabilities that may be at work are the decorrelation instability (Lilly 1983) and the zig-zag instability (Billant \& Chomaz 2000) both of which act on vertically coherent flow regions or vortices and break this coherence leading to the formation of thinner layers.

Another problem in which there is symmetry breaking along an axis is the case of rotating turbulence. This turbulent flow is known to present a number of analogies with stratified turbulence (Davidson 2013), and compared to the stratified case in which the flow is dominated by layered structures, the rotating case is also dominated by anisotropic structures, but here they are elongated along the axis of rotation. It is also well-known that these elongated structures are preferentially cyclonic rather than anticyclonic, and hence preferentially spin in the same sense as the system rotation (see Hopfinger et al. 1982; Bartello et al. 1994). In mathematical terms, this translates in the vertical vorticity $\omega_{z}$ being mostly positive for large values of $\left|\omega_{z}\right|$ and hence to this quantity having non-zero positive skewness (if the rotation $\Omega$ is in the positive vertical direction). The skewness of $\omega_{z}$ has been measured in a number of experiments (Staplehurst et al. 2008; Gan et al. 2016) and numerical simulations (Bartello et al. 1994; Deusebio et al. 2014) of rotating turbulence, and typical values are of the order of unity. The explanation put forward by Bartello et al. (1994) for the cyclone-anticyclone asymmetry in rotating turbulence is that anticyclones are more susceptible to becoming unstable to the centrifugal instability than cyclones, at a Rossby number of order unity. Thus, anticyclones would be depleted by the centrifugal instability and cyclones would remain stable, leading to the asymmetry.

A theoretical explanation for the creation of strong positive vorticity of the same sign as $\boldsymbol{\Omega}$ in rotating turbulence has been suggested more recently by Gence \& Frick (2001). In their study, they considered first homogeneous isotropic turbulence in which the skewness of $\omega_{z}$ is zero and then tried to answer the question of what would happen if the system rotation is suddenly switched on at $t=0$. Focusing on the equation for the evolution of the "dimensional skewness" of $\omega_{z},\left\langle\omega_{z}^{3}\right\rangle$, Gence \& Frick were able to show that this reduces to, at the instant after rotation is applied:

$$
\frac{d}{d t}\left\langle\omega_{z}^{3}\right\rangle=\frac{2}{5} \Omega\left\langle\omega_{i} \omega_{j} S_{i j}\right\rangle
$$

where $\Omega=\Omega \cdot \mathbf{e}_{z}$ and $S_{i j}$ is the strain rate tensor. The reason why equation 1.2 is valid 
at the first instant after $t=0$ is that all the other terms are zero since the turbulence is still isotropic. Considering then that the quantity $\left\langle\omega_{i} \omega_{j} S_{i j}\right\rangle$ is positive in homogeneous isotropic turbulence, Gence \& Frick used equation 1.2 to show that, if $\Omega>0$, the vertical vorticity skewness becomes positive as a result of the application of a system rotation $\boldsymbol{\Omega}$. This theoretical argument may explain why there is an inherent tendency for rotating turbulence to develop vorticity of the same sign as the rotation, and so create prevalence of cyclones over anticyclones in this flow.

In the present study we consider if similar theoretical arguments as those used by Gence \& Frick for rotating turbulence, can be used in stratified turbulence to show that the vertical buoyancy gradient has a tendency to become positive for high values of $|\partial b / \partial z|$ and so to develop a positive skewness. By extension, we are looking for a natural tendency of stratified turbulence towards layering of the density and buoyancy fields.

\section{Theoretical considerations}

\subsection{Response of vertical buoyancy gradient to a stable stratification}

We consider a similar thought experiment to the one considered by Gence \& Frick (2001) for rotating turbulence, which we briefly summarized previously. We still consider the case of homogeneous isotropic turbulence but now in the presence of an advected passive scalar. We focus on the case of an initially decaying scalar field, but the analysis can be extended to the case of a homogeneous and isotropic source or forcing of the scalar. In this thought experiment, the passive scalar is advected in a field of fully developed turbulence until, at $t=0$, a linear background stratification of the scalar field as well as gravity are suddenly "switched on". This means that the scalar in question, which we denote as $b$, suddenly ceases being a passive scalar and becomes an active scalar at $t=0$, i.e. at this time the scalar effectively becomes the buoyancy in a linearly stratified turbulent flow. Note that the arguments described here can also be applied to the case of a passive scalar which remains passive for all times and in which a mean scalar gradient is suddenly applied at $t=0$. Hence, the result can provide insight also into the observation of non-zero scalar gradient skewness and staircase profiles in the case of passive scalar advection with a background scalar gradient (Warhaft 2000).

After the stratification is switched on, the buoyancy behaves according to the following equation:

$$
\frac{\partial b}{\partial t}+\mathbf{u} \cdot \nabla b=-N^{2} w+\mathcal{D} \nabla^{2} b
$$

where $\mathbf{u}=\left[\begin{array}{lll}u & v & w\end{array}\right]$ is the velocity field and $\mathcal{D}$ is the buoyancy diffusivity. In order to consider the vertical buoyancy gradient, we take the $z$-derivative of this equation to obtain the evolution equation for $\partial b / \partial z$ :

$$
\frac{\partial}{\partial t}\left(\frac{\partial b}{\partial z}\right)+\mathbf{u} \cdot \nabla\left(\frac{\partial b}{\partial z}\right)=-N^{2} \frac{\partial w}{\partial z}-\frac{\partial \mathbf{u}}{\partial z} \cdot \nabla b+\mathcal{D} \nabla^{2}\left(\frac{\partial b}{\partial z}\right)
$$

which can be found using the identity $\partial / \partial z(\mathbf{u} \cdot \nabla b)=\partial \mathbf{u} / \partial z \cdot \nabla b+\mathbf{u} \cdot \nabla(\partial b / \partial z)$. This equation is an advection-diffusion equation with two additional terms. The first term on the RHS represents a change in $\partial b / \partial z$ due to the vertical strain of the vertical velocity acting on the background buoyancy gradient. The second term on the RHS represents creation/destruction of vertical buoyancy gradient due to tilting of the overall buoyancy gradient by the vertical shear. 
We proceed and multiply equation 2.2 by $3(\partial b / \partial z)^{2}$ to obtain, after some rearrangement, the following evolution equation for $(\partial b / \partial z)^{3}$ :

$$
\begin{aligned}
\frac{\partial}{\partial t}\left(\frac{\partial b}{\partial z}\right)^{3} & +\mathbf{u} \cdot \nabla\left(\frac{\partial b}{\partial z}\right)^{3}= \\
& -3 N^{2} \frac{\partial w}{\partial z}\left(\frac{\partial b}{\partial z}\right)^{2}-3\left(\frac{\partial b}{\partial z}\right)^{2}\left(\frac{\partial \mathbf{u}}{\partial z} \cdot \nabla b\right)+\mathcal{D} \nabla^{2}\left(\frac{\partial b}{\partial z}\right)^{3} \\
& -6 \mathcal{D} \frac{\partial b}{\partial z}\left[\nabla\left(\frac{\partial b}{\partial z}\right) \cdot \nabla\left(\frac{\partial b}{\partial z}\right)\right]
\end{aligned}
$$

The last step consists in taking the ensemble average of equation 2.3 to obtain an equation for the "dimensional skewness", $\left\langle(\partial b / \partial z)^{3}\right\rangle$. Before doing this, we observe that by setting $N=0$ in equation 2.3 we obtain the governing equation valid in the absence of stratification, for $t<0$, when $b$ is a passive scalar. It is clear that ensemble averaging this equation would result in a number of terms such as:

$$
\left\{\begin{array}{l}
\left\langle\mathbf{u} \cdot \nabla\left(\frac{\partial b}{\partial z}\right)^{3}\right\rangle=C \\
3\left\langle\left(\frac{\partial b}{\partial z}\right)^{2}\left(\frac{\partial \mathbf{u}}{\partial z} \cdot \nabla b\right)\right\rangle=D
\end{array}\right.
$$

where $C$ and $D$ are constants which do not depend on spatial position within the turbulent flow. This follows from homogeneity of the turbulence. Now, because we assume zero net boundary flux it follows from incompressibility that $C=0$ and because of isotropy of the turbulent flow for $t<0$ it is also true that $D=0$. To confirm this, one can verify that the second equation in the set of equations 2.4 would change sign under a reflection of the $z$-axis which is impossible for an isotropic flow. Hence the only possibility is that $D=0$. Using similar arguments, one can confirm that the two remaining terms involving diffusivity are also zero on average. This means that $\left\langle(\partial b / \partial z)^{3}\right\rangle=0$ for $t<0$ and the vertical buoyancy gradient has zero skewness while there is no stratification. This fact can also be directly explained by realizing that $\left\langle(\partial b / \partial z)^{3}\right\rangle$ needs to be zero if the scalar field is isotropic.

The above picture changes when the stratification is introduced. Considering the above discussion, ensemble averaging of equation 2.3 leaves us with, at $t=0^{+}$:

$$
\frac{d}{d t}\left\langle\left(\frac{\partial b}{\partial z}\right)^{3}\right\rangle=-3 N^{2}\left\langle\frac{\partial w}{\partial z}\left(\frac{\partial b}{\partial z}\right)^{2}\right\rangle
$$

First of all, it is important to apply the above arguments and let $\left\langle\partial w / \partial z(\partial b / \partial z)^{2}\right\rangle=E$, which allows us to confirm that the constant $E$ is a priori non-zero in isotropic turbulence with a passive scalar, since $z$-reflection maintains its sign. Now, since $\left\langle(\partial b / \partial z)^{3}\right\rangle=0$ at $t=0$, the above equation shows that the stratification will cause the formation of a nonzero skewness, and this occurs instantaneously after the application of $N$. It remains to determine of what sign the skewness of $\partial b / \partial z$ will become. To understand this we need to know the sign of $E$. The term under scrutiny is closely related to the velocity-scalar third-order structure function, $D_{L b b}(r)=\left\langle\Delta u(\Delta b)^{2}\right\rangle$, as we will now see. Here $r$ is the separation distance of the two-point statistics and $\Delta u$ is a longitudinal velocity difference. There is an exact relationship in homogeneous isotropic turbulence for the velocity-scalar third-order structure function, known as Yaglom's relation (Yaglom 1949), 


$$
\left\langle\Delta u(\Delta b)^{2}\right\rangle=-\frac{4}{3} \chi r \quad \text { for } \quad \eta_{b} \ll r \ll L
$$

where $\chi$ is the dissipation rate of half the scalar variance, $\chi=\mathcal{D}\langle\nabla b \cdot \nabla b\rangle$. The Batchelor scale $\eta_{b}$ corresponds to the smallest scale of the scalar field, while $L$ is an integral scale of the turbulence. This relation is valid in what is called the convective-inertial range and the negative sign of $\left\langle\Delta u(\Delta b)^{2}\right\rangle$ in this range is related to the flux of scalar variance from large scales to small scales. The fact that $\left\langle\Delta u(\Delta b)^{2}\right\rangle<0$ results in this flux being positive, indicating a forward cascade of scalar variance.

For small separations $r$, we can follow the approach of Yeung et al. (2002) and use first-order Taylor expansions to let the scalar-velocity structure function be approximated by:

$$
\left\langle\Delta u(\Delta b)^{2}\right\rangle \approx\left\langle\frac{\partial w}{\partial z}\left(\frac{\partial b}{\partial z}\right)^{2}\right\rangle r^{3}
$$

where we have let the structure function separation be in the $z$-direction. This is exactly the term we need to consider. It thus follows that this term is negative since the velocityscalar structure function is negative for all $r$. This feature of $\left\langle\Delta u(\Delta b)^{2}\right\rangle$ has been observed in several studies of passive scalar advection in isotropic turbulence with or without mean scalar gradient (Yeung et al. 2002; Watanabe \& Gotoh 2004; de Bruyn Kops 2015). It is simply a consequence of the fact that the direction of the cascade of scalar variance is always downscale towards the dissipative scales, even at small $r$.

Taking into account this result that $E<0$, equation 2.5 means that the vertical buoyancy gradient becomes positively skewed at $t=0^{+}$. Hence, the effect of the stable stratification on an isotropic field of turbulence with a scalar $b$ that suddenly becomes influenced by gravity is to create a positive skewness of $\partial b / \partial z$. Effectively, as argued by Warhaft (2000) in the context of passive scalar advection, this means that the buoyancy field instantly becomes anisotropic since reflection of the $z$-axis no longer leaves all terms of equation 2.3 invariant (when averaged).

\subsection{Symmetry of Boussinesq set of equations in a linearly stratified fluid}

The above idealization of the problem evidences an inherent tendency of stratified turbulence towards symmetry breaking in the vertical direction, and towards the development of a positive skewness of the vertical buoyancy gradient, $S$. It is worth inspecting the Boussinesq set of equations that governs this turbulent flow and to consider its symmetry with respect to the $z$-axis. The full set of equations for a linearly stratified flow under the Boussinesq approximation is:

$$
\begin{aligned}
\frac{\partial \mathbf{u}}{\partial t}+\mathbf{u} \cdot \nabla \mathbf{u} & =-\frac{1}{\rho_{0}} \nabla p+b \mathbf{e}_{z}+\nu \nabla^{2} \mathbf{u} \\
\frac{\partial b}{\partial t}+\mathbf{u} \cdot \nabla b & =-N^{2} w+\mathcal{D} \nabla^{2} b \\
\nabla \cdot \mathbf{u} & =0
\end{aligned}
$$

where $\nu$ is the viscosity. The pressure $p$ is a perturbation pressure away from the hydrostatic pressure profile due to the linear background density profile. Equations 2.8 and 2.10 are the momentum and continuity equations. They are both symmetric with respect to reflections of the $z$-axis, in a statistical sense. Focusing for example on the $z$ component of the momentum equation, it is clear that the vertical velocity $w$ and hence 
also $\partial w / \partial t$ change sign at every point under a $z$-reflection but so do all other terms in this equation, and so statistics of $w$ remain unchanged in the absence of mean flow. On the other hand, consideration of the buoyancy equation immediately reveals that all terms remain of the same sign, except for $-N^{2} w$, which changes sign. This is because $N$ is a constant in the equations. Thus, the buoyancy equation is asymmetric in the vertical direction. The asymmetry of the vertical buoyancy gradient and its positive skewness are just consequences of this basic property of the buoyancy equation. In some sense this asymmetry of the governing equations is reassuring because it implies, loosely speaking, that stratified turbulence knows that gravity is pointing downwards (and not upwards!).

Finally, it is worth pointing out that since equations 2.8-2.10 are coupled there may be an effect of the asymmetry of $\partial b / \partial z$ on the statistics of $\mathbf{u}$ and its gradients. In particular, the buoyancy will have a direct effect on the vertical velocity, which will in turn affect the horizontal components of the velocity. However, since the direct effects are felt by $w$ which is small in strongly stratified turbulence, it is expected that the overall effect on u will be weak.

\subsection{Scaling prediction for the skewness}

\subsubsection{Stratified turbulence}

Having described the behaviour of the vertical buoyancy gradient skewness, we now look for an estimate of this quantity. From equation 2.5, which is the main result of the above analysis, we can make some steps forward by assuming that $\left\langle\partial w / \partial z(\partial b / \partial z)^{2}\right\rangle \approx$ const up to a certain time $\tau$ after the application of the stratification. This gives an estimate for the dimensional skewness (which we denote as $B_{z 3}$ from this point onwards),

$$
B_{z 3} \equiv\left\langle\left(\frac{\partial b}{\partial z}\right)^{3}\right\rangle \approx-3 N^{2} \tau\left\langle\frac{\partial w}{\partial z}\left(\frac{\partial b}{\partial z}\right)^{2}\right\rangle
$$

For our estimate of $B_{z 3}$ to be reliable, the second hypothesis which must hold is that most of the skewness of the "fully developed" stratified turbulence is created in this initial adjustment period. In their work, Gence \& Frick (2001) assumed the relevant timescale for turbulence subjected suddenly to solid-body rotation to be $\tau=\tau_{k}$, where $\tau_{k}=(\nu / \epsilon)^{1 / 2}$ is the Kolmogorov timescale. In the present case of strongly stratified turbulence we use a different timescale. We expect the vertical scales of the layer-interface structure to be significantly larger than the Kolmogorov lengthscale, $\eta$. Since the steps observed by Holford \& Linden (1999); Kimura et al. (2016) are sharp we expect this to be true even for the vertical scale of the interfaces. As a result, we envisage that a significantly larger timescale than $\tau_{k}$ is relevant. Moreover, the fact that the stratification is strong implies that the horizontal Froude number is small, $F r_{h} \ll 1$. The horizontal Froude number is given by $\mathrm{Fr}_{h}=u_{h} /\left(N \ell_{h}\right)$, where $u_{h}$ is a typical velocity scale of the horizontal motions and $\ell_{h}$ is a horizontal lengthscale. Hence, it is clear that there are two natural timescales in this problem, whose ratio is equal to $F r_{h}$. The first timescale is $N^{-1}$, the buoyancy timescale, and the second timescale is $\ell_{h} / u_{h}$, the horizontal eddy turnover time. Since $\mathrm{Fr}_{h} \ll 1$, we have $N^{-1} \ll \ell_{h} / u_{h}$, meaning that the buoyancy timescale is much smaller than the non-linear timescale. Considering that we expect the turbulence to adjust to the imposition of the stratification on a fast timescale over which equation 2.11 holds, we set $\tau \sim N^{-1}$. Note that with this choice of timescale, $\tau / \tau_{k} \sim \epsilon^{1 / 2} /\left(\nu^{1 / 2} N\right)=R e_{b}^{1 / 2} \gg 1$ since $R e_{b} \gg 1$ in strongly stratified turbulence. Hence, the scaling for the dimensional skewness is: 


$$
B_{z 3} \sim N\left\langle\frac{\partial w}{\partial z}\left(\frac{\partial b}{\partial z}\right)^{2}\right\rangle \sim S_{u b} N\left\langle\left(\frac{\partial w}{\partial z}\right)^{2}\right\rangle^{1 / 2}\left\langle\left(\frac{\partial b}{\partial z}\right)^{2}\right\rangle
$$

in which we have followed Yeung et al. (2002) and introduced the quantity $S_{u b}$ defined as $S_{u b} \equiv\left\langle\partial w / \partial z(\partial b / \partial z)^{2}\right\rangle /\left[\left\langle(\partial w / \partial z)^{2}\right\rangle^{1 / 2}\left\langle(\partial b / \partial z)^{2}\right\rangle\right]$. This quantity was found to be $S_{u b} \sim 1$ (and its sign was negative) in the DNS of turbulence in the presence of a passive scalar with a mean scalar gradient of Yeung et al. (2002). For the present case we will also assume $S_{u b} \sim 1$. Furthermore, we use the isotropic turbulence relations for the spatial gradients, which leads to:

$$
B_{z 3} \sim N \sqrt{\frac{\epsilon}{15 \nu}} \frac{\chi}{3 \mathcal{D}}
$$

where $\epsilon$ is the kinetic energy dissipation rate, $\epsilon=2 \nu\left\langle S_{i j} S_{i j}\right\rangle$. In stratified turbulence, the buoyancy $b$ is related to the potential energy $E_{P}=\left\langle b^{2}\right\rangle /\left(2 N^{2}\right)$, which is actually a surrogate for the available potential energy in this flow. The related potential energy dissipation is $\epsilon_{p}=\chi / N^{2}$. Substitution of this expression in equation 2.13 gives, after dropping the numeric constants,

$$
B_{z 3} \sim \sqrt{\frac{\epsilon}{\nu}} \frac{\epsilon_{p} N^{3}}{\mathcal{D}}
$$

We make our final assumption that $\epsilon_{p} \sim \epsilon$, a scaling which is valid for strongly stratified turbulence, a regime in which the mixing efficiency $\Gamma=\epsilon_{p} / \epsilon$ is found theoretically and numerically to be a constant of order unity, $\Gamma \sim 1$ (Maffioli et al. 2016). This is a strong assumption since the scaling for the mixing efficiency is in theory only valid when the stratified turbulence is in a fully developed state, and not in a transient adjustment phase. Nonetheless, we proceed and with this assumption we obtain the following scaling expression for the dimensional skewness:

$$
B_{z 3} \sim \frac{\epsilon^{3 / 2} N^{3}}{\nu^{1 / 2} \mathcal{D}}
$$

If this scaling for $B_{z 3}$ holds we can obtain a scaling for the vertical buoyancy gradient skewness $S$. Referring back to equation 1.1, and using the isotropic relation for the vertical buoyancy gradient variance, which we will from this point denote as $B_{z 2} \equiv\left\langle(\partial b / \partial z)^{2}\right\rangle$, we obtain:

$$
S \sim \frac{\frac{\epsilon^{3 / 2} N^{3}}{\nu^{1 / 2} \mathcal{D}}}{\left(\frac{\epsilon_{p} N^{2}}{\mathcal{D}}\right)^{3 / 2}} \sim \operatorname{Pr}^{-1 / 2}
$$

where $\operatorname{Pr}=\nu / \mathcal{D}$ is the Prandtl number. The case of non-unity Prandtl number may be relevant for ocean turbulence where $\operatorname{Pr}$ (or actually the Schmidt number in this case) is between $O(10)$ and $O\left(10^{3}\right)$. On the other hand, if we set $\operatorname{Pr}=1$, we can obtain a simplified expression for the dimensional skewness,

$$
B_{z 3} \sim \frac{\epsilon^{3 / 2} N^{3}}{\nu^{3 / 2}}
$$

and for the skewness, $S$, 


$$
S \sim 1
$$

A Prandtl number of unity is relevant for stratified turbulence in the atmosphere, where $\operatorname{Pr} \sim 1$, and this is the value used in the present numerical simulations. With this choice, we predict the vertical buoyancy gradient skewness in strongly stratified turbulence to be of order unity, $S \sim 1$. Both this result and the expression for $B_{z 3}$, equation 2.17, can be tested using DNS, and this will be the subject of the following section.

The main assumption of the scaling analysis leading to equations 2.17 and 2.18 is that the relevant timescale for the generation of positive skewness of the vertical buoyancy gradient is the fast timescale $\tau \sim N^{-1}$. It is important to note that this timescale is of the order of the period of linear internal gravity waves, which will be generated at $t=0^{+}$ and at subsequent times. As a result $\tau \sim N^{-1}$ is often thought of as a linear timescale (Riley \& Lelong 2000). If the analysis is appropriate for this problem and so if the above expressions for the skewness turn out to be accurate, one could interpret this result as being due to the action of internal gravity waves. It is plausible that, at least in our thought experiment, the fast propagation of the waves generated at $t=0^{+}$could create a skewness in the initially isotropic scalar field. By extension, the waves may be playing a role in setting up the staircase profile of the buoyancy field.

\subsubsection{Passive scalar advection}

We consider a similar analysis of equation 2.5 for the case in which $b$ is a passive scalar. For consistency with the literature on passive scalar advection, we change the symbol of the scalar in this section to $\phi \equiv b$. As we saw in the previous section, the most important choice of the analysis was the choice of the timescale and we expect the same to be true in the present case. We again depart from Gence \& Frick (2001) who considered the Kolmogorov timescale to be relevant in the case of rotating turbulence. It is believed that the approximate relation for the third moment of the vertical scalar gradient, see equation 2.19 below, is again valid for a significantly longer time. In saying this we follow Bos (2014), who proposed that the relevant timescale of thirdorder statistics of passive scalars in turbulence in the presence of a mean scalar gradient is the integral timescale. Using the integral timescale, $T$, in triadic closure equations as well as in a dimensional analysis of the problem, Bos (2014) was able to recover a vertical scalar gradient skewness, $S_{\phi z}=O(1)$, in agreement with experiments and simulations (Mydlarski \& Warhaft 1998; Yeung et al. 2002). It is interesting to test if using $\tau \sim T$ will give similar results within the present analysis. In passive scalar advection the turbulent flow is isotropic and the integral lengthscale of the scalar field is determined by the large eddies and so is of the same order of magnitude as the integral lengthscale of the turbulence, $L_{\phi} \sim \Phi /(d \bar{\phi} / d z) \sim L$, where $\Phi=\left\langle\phi^{2}\right\rangle^{1 / 2}$. The integral timescale is thus $T \sim L / u \sim \Phi /(u d \bar{\phi} / d z)$. Using this timescale in the "initial adjustment" approximation of equation 2.5 we obtain $\left(\phi_{z 3}\right.$ is the dimensional skewness of $\left.\partial \phi / \partial z\right)$ :

$$
\phi_{z 3} \sim \frac{d \bar{\phi}}{d z} T\left\langle\frac{\partial w}{\partial z}\left(\frac{\partial \phi}{\partial z}\right)^{2}\right\rangle \sim \frac{\Phi \chi \epsilon^{1 / 2}}{u \mathcal{D} \nu^{1 / 2}}
$$

To proceed we use the asymptotic finite limits reached by kinetic energy and scalar dissipation rates at high Reynolds number, $\epsilon \sim u^{3} / L$ and $\chi \sim \Phi^{2} u / L$ (see Donzis et al. 2005). Hence, the expression for $\phi_{z 3}$ simplifies to:

$$
\phi_{z 3} \sim \frac{\chi^{3 / 2}}{\mathcal{D} \nu^{1 / 2}}
$$


Finally we use the isotropic scaling for the vertical scalar gradient variance, $\phi_{z 2} \sim \chi / \mathcal{D}$, to arrive at an estimate for the vertical scalar gradient skewness, $S_{\phi z}$ :

$$
S_{\phi z} \sim \frac{\left(\frac{\chi^{3 / 2}}{\mathcal{D} \nu^{1 / 2}}\right)}{\left(\frac{\chi^{3 / 2}}{\mathcal{D}^{3 / 2}}\right)} \sim \frac{\mathcal{D}^{1 / 2}}{\nu^{1 / 2}}=1
$$

in the case $\operatorname{Pr}=1$. Hence, the scaling analysis predicts a skewness of order unity, similarly to the case of stratified turbulence. This result is in agreement with previous studies on passive scalar advection at $\operatorname{Pr} \sim 1$ (Mydlarski \& Warhaft 1998; Yeung et al. 2002). It is of interest to also consider the case $\operatorname{Pr} \neq 1$, which, as before, results in the following scaling for the skewness:

$$
S_{\phi z} \sim \operatorname{Pr}^{-1 / 2}
$$

and so the skewness is predicted to decrease with increasing values of $\mathrm{Pr}$. Two previous numerical studies focusing on the skewness of passive scalar gradients have considered non-unity Prandtl number cases over a large range of $\operatorname{Pr}$ (Yeung et al. 2002; Schumacher \& Sreenivasan 2003) and they both found a weakly decreasing skewness with increasing $\operatorname{Pr}$, qualitatively in agreement with equation 2.22. This result may have an important implication since it predicts that at high $\operatorname{Pr}$ the skewness $S_{\phi z} \rightarrow 0$, meaning that the scalar field becomes isotropic. The reduction of the scalar anisotropy up to relatively high $\operatorname{Pr}$ for what is attainable by numerical simulations, $\operatorname{Pr}=64$, was confirmed by the results of Schumacher \& Sreenivasan (2003).

\section{Numerical simulations}

\subsection{Description of DNS simulations}

A series of DNS of forced stratified turbulence was carried out, starting from randomphase initial conditions for $\mathbf{u}$ and $b=0$ at all points in the case of the buoyancy. The exception is the highest resolution run (run R4) which was initialized using a snapshot of another run (run R3). This means that the configuration of the simulations is very different from the thought experiment described in $\S 2$ but it was chosen for numerical convenience and may lead to a similar structure of stratified turbulence, for which equations 2.17-2.18 are still applicable. The Boussinesq set of equations was solved numerically using a pseudo-spectral method, with an algorithm based on Rogallo's method (Rogallo 1981). An artificial body force, f, was added to the RHS of the momentum equation, equation 2.8 , to obtain a statistically stationary field of stratified turbulence. In total six runs were performed, with two significantly different forcing schemes. In four of the runs, the forcing was applied to the vortical component of the velocity field only and this was done only for vertical wavenumbers $k_{z}=0$ so that $\mathbf{f}$ points in the horizontal direction and does not change along the vertical (this forcing is very similar to that of Kimura et al. 2016). The forcing was concentrated in horizontal wavenumbers in a circular shell at $k_{h}=\sqrt{k_{x}^{2}+k_{y}^{2}}=3$. For more details on these four DNS simulations, the forcing scheme and the choice of box aspect ratio, the reader is referred to our previous study, Maffioli (2017). For the final two DNS runs the well-known stochastic forcing scheme of Eswaran \& Pope was used (see Eswaran \& Pope 1988). Unlike the forcing scheme used in the other simulations, this forcing is isotropic and so equally forces the vortex and the wave component of the flow. Another important difference is 
that the forcing scheme of the first four runs is white noise in time (uncorrelated in time), while the Eswaran \& Pope stochastic forcing has a finite correlation timescale. In our application of Eswaran \& Pope forcing, the force is applied in Fourier space for all modes within the sphere of radius $|\mathbf{k}|=3$, where $\mathbf{k}=\left[k_{x} k_{y} k_{z}\right]$ is the wavevector.

Time advancement is performed with a second-order Runge-Kutta scheme and dealiasing of the advective terms is obtained using a combination of truncation and phase shifting (see Rogallo 1981). Since phase shifting is used, only partial truncation is employed, with truncation of modes with $|\mathbf{k}|>k_{\max }=\sqrt{2} / 3\left(2 \pi / L_{x}\right) N_{x}$. The number of grid points in the three directions is given by $N_{x}, N_{y}, N_{z}$, and the box dimensions are $L_{x}, L_{y}, L_{z}$. In all runs $L_{x}=L_{y}=2 \pi$, while in some runs the vertical box dimension, $L_{z}$, is smaller than $L_{x}$ to reduce the computational cost, taking advantage of the anisotropy of stratified turbulence. The grid spacing is uniform in all three directions. A list of computational and physical parameters of the runs is given in table 1 . The vertical box dimension for each run is given by $L_{z}=L_{x}\left(N_{z} / N_{x}\right)=2 \pi\left(N_{z} / N_{x}\right)$. The good resolution of the smallest scales is achieved for the DNS runs, all of which have $k_{\max } \eta \approx 1.5$ during the statistically stationary period. The Prandtl number is unity for all simulations.

Our working definitions of the horizontal Froude number, buoyancy Reynolds number and Reynolds number are $F r_{h} \equiv \epsilon /\left(N u_{h}^{2}\right), R e_{b} \equiv \epsilon /\left(\nu N^{2}\right)$ and $R e \equiv u_{h}^{4} /(\nu \epsilon)$, respectively. These relations make use of the dissipation scaling expected to hold in strongly stratified turbulence, $\epsilon \sim u_{h}^{3} / \ell_{h}$ (Lindborg 2006). In this way we avoid the explicit use of a lengthscale in the definitions of $\mathrm{Fr}_{h}$ and $R e$. This makes the values of the Froude number and Reynolds number easier to compare with other works, considering there are several definitions of the integral lengthscale used in turbulence studies. Note that the definition of the horizontal velocity scale used here is $u_{h}=\sqrt{\left\langle u^{2}+v^{2}-u_{s m}^{2}-v_{s m}^{2}\right\rangle / 2}$, where $1 / 2\left\langle u_{s m}^{2}+v_{s m}^{2}\right\rangle$ is the kinetic energy contained in the "shear modes", the horizontal box-filling motions (i.e. modes with horizontal wavenumbers $k_{x}=k_{y}=0$ ). The kinetic energy in the shear modes continues to grow over the course of a run and they appear to not participate in the transfer of energy to small scales. Since Eswaran \& Pope forcing directly excites the shear modes, the kinetic energy at the end of runs R5ep and R6ep is dominated by these modes and hence it was decided to remove their contribution to $u_{h}$. For consistency this procedure was repeated for all runs. Note also that in the simulation work presented in this section, the symbol $\langle\ldots\rangle$ changes meaning and denotes a volume average over the numerical domain but because of ergodicity this should not have an effect on the results. In terms of the stratified turbulence regimes, these simulations have $F_{h}=O\left(10^{-2}\right)$ and $R e_{b}=O(10)$ and so they are within, or at least close to being within, the strongly stratified turbulence regime, which requires $F_{h} \ll 1$ and $\operatorname{Re}_{b} \gg 1$ (see, e.g., Brethouwer et al. 2007). The exception to these parameter magnitudes is run R6ep which has a significantly higher buoyancy Reynolds number than the other runs, $R e_{b}=64.1$, and maintains a relatively low Froude number. In table 1, the value of the mixing efficiency $\Gamma=\epsilon_{p} / \epsilon$, with $\epsilon$ and $\epsilon_{p}$ time averaged over the steady state period, is given for each run. From these values it is possible to see that indeed $\Gamma \sim 1$ and in particular that $\Gamma$ is approximately constant across the runs, lying within the narrow range $\Gamma=0.3-0.45$.

\subsection{Asymmetry in the visualisations and vertical profiles of buoyancy}

In this section we focus on the run at highest resolution, run R4, and present visualisations and vertical profiles taken during the steady state period. In figure 2, visualisations of a horizontal component of velocity, $u$, and of buoyancy in a vertical slice through the centre of the domain are shown. It is possible to appreciate the anisotropic structure of stratified turbulence, composed of horizontal layers in both the velocity and buoyancy. 


$\begin{array}{lccccccc}\text { Run } & N_{x}=N_{y} & N_{z} & F_{h} & R e_{b} & R e\left(\times 10^{3}\right) & \Gamma & \text { Forcing } \\ \text { R1 } & 1024 & 512 & 0.051 & 13.6 & 5.2 & 0.39 & \text { Vortical mode } \\ \text { R2 } & 2048 & 512 & 0.031 & 14.1 & 14.6 & 0.38 & \text { Vortical mode } \\ \text { R3 } & 4096 & 1024 & 0.024 & 17.0 & 29.1 & 0.31 & \text { Vortical mode } \\ \text { R4 } & 8192 & 2048 & 0.017 & 21.7 & 77.8 & 0.31 & \text { Vortical mode } \\ \text { R5ep } & 1024 & 1024 & 0.047 & 16.8 & 7.6 & 0.40 & \text { Eswaran \& Pope } \\ \text { R6ep } & 2048 & 2048 & 0.054 & 64.1 & 22.1 & 0.44 & \text { Eswaran \& Pope }\end{array}$

TABLE 1. List of DNS simulations including relevant physical parameters. These parameters are obtained using time-averaged physical quantities over the statistically stationary period of the simulation.

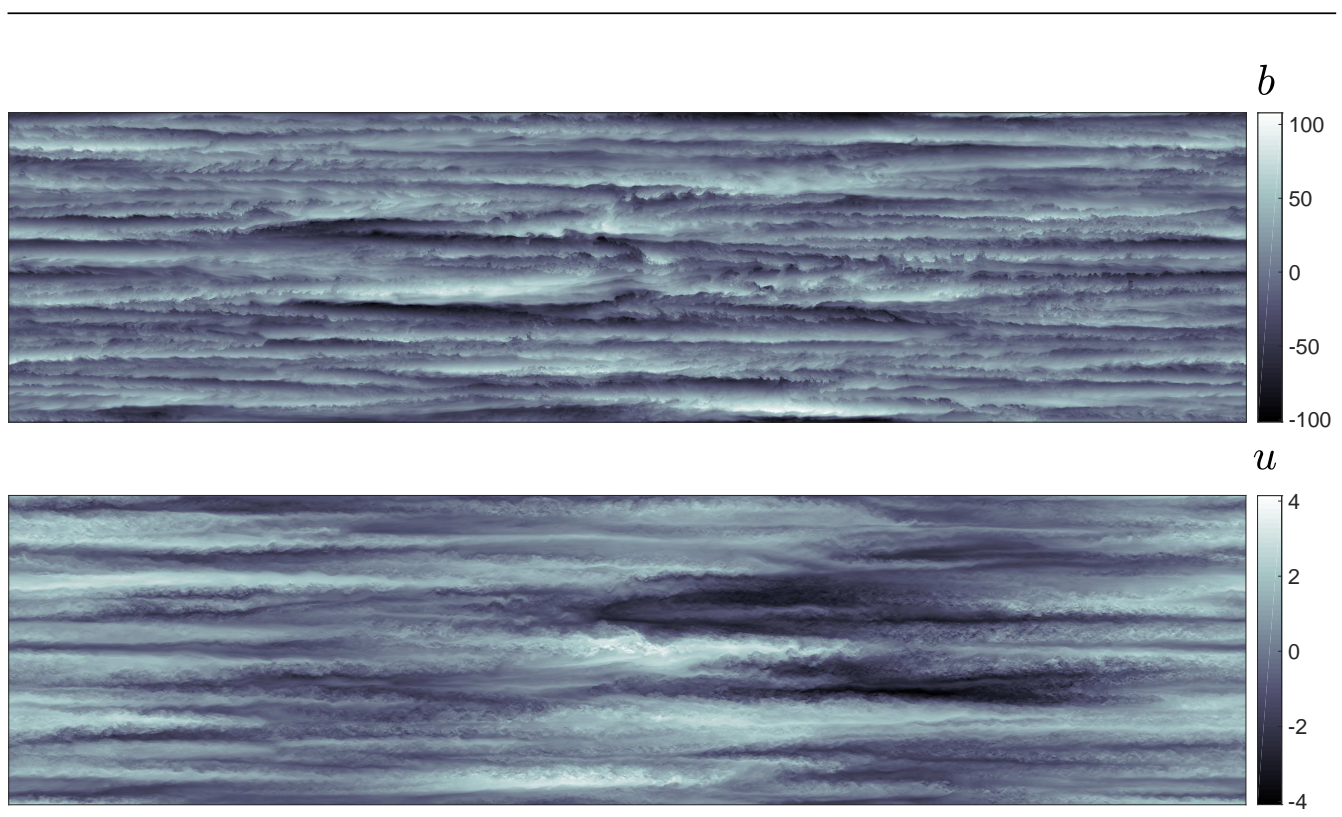

FiguRE 2. Visualisation of $u$ and $b$ in a vertical $x-z$ plane taken through the centre of the domain in the final snapshot of run R4, at the last time instant of this run.

The complexity and three-dimensionality of the turbulence in this high-Re simulation is also evident, with a number of instabilities seemingly taking place at the interface between layers, which are sometimes slightly inclined to the horizontal. This can be seen in more detail in the zoom-in of the same vertical slice, presented in figure 3.

We now assess the symmetry of the velocity and buoyancy fields. It should be relatively clear from figures 2-3 that the horizontal velocity component is approximately symmetric with respect to reflection of the $z$-axis, in agreement with our previous considerations. On the other hand, it can be clearly observed that the buoyancy field is asymmetric with respect to $z$-reflection. Taking a point at the bottom of the visualisations of $b$ and moving upwards, it is possible to see that positive changes in $b$ (from black to white) occur rather abruptly while negative changes in $b$ (from white to black) are more gradual. This means positive $\partial b / \partial z$ tends to be stronger in magnitude than negative $\partial b / \partial z$. We have hence confirmed, for now in a qualitative way, that the vertical gradient of the buoyancy field indeed becomes positively skewed in DNS of stratified turbulence. To go back to figures 2-3, it appears that the visualisations of $u$ could be turned upside down without 

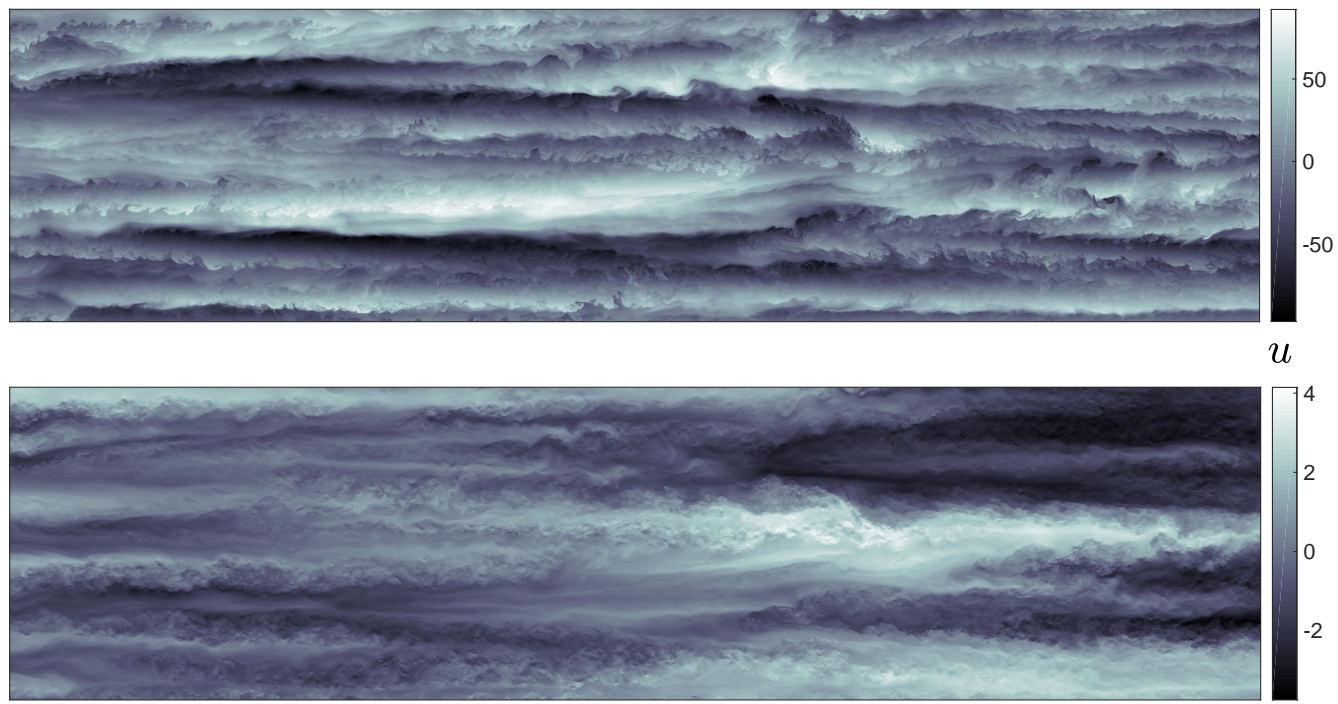

FiguRE 3. Zoom-in of a portion of the vertical slices in figure 2.

significant change but the same is not true for the visualisations of $b$, which would be different.

Are vertical profiles of buoyancy in the DNS similar to the staircase profile shown in figure 1? To answer this question we need to consider vertical profiles of total buoyancy, $b_{\text {tot }}=b+N^{2} z$, in the DNS. Figure 4 shows four vertical buoyancy profiles sampled throughout the domain, again in a snapshot of run R4 during steady state. While strongly stable interfaces adjacent to almost perfectly mixed layers are frequently observed, a regular staircase profile is not observed (in agreement with the discussion of Gregg et al. 2018). Layer-interface segments are often isolated and not in periodic succession as in figure 1. In fact, large portions of the profiles are irregular and do not deviate significantly from the mean stratification, indicating a more complicated picture. Nonetheless, it is clear that the buoyancy profiles shown in figure 4 should lead to a positive vertical buoyancy gradient skewness.

\subsection{PDFs of buoyancy gradient}

A good instrument to check if a quantity has asymmetric statistics is, of course, its PDF. As is well known, all the moments of a quantity - mean, variance, skewness and higher order moments - can be obtained from this single curve. Our focus is the vertical buoyancy gradient and so we consider its PDF and, for comparison, also that of the horizontal buoyancy gradients. In figure 5, the average of the PDFs of the two horizontal gradients of buoyancy and the PDF of the vertical gradient are given, both at the final snapshot of run R4. The horizontal buoyancy gradients can be seen to be approximately symmetric with a low non-dimensional skewness; this PDF does not compare well with the Gaussian PDF (not shown) of the same standard deviation, $\sigma$, as the tails of the average PDF of $\partial b / \partial x$ and $\partial b / \partial y$ are significantly wider. Turning to the PDF of $\partial b / \partial z$, important differences are evident: the PDF of $\partial b / \partial z$ is clearly asymmetric and is skewed towards positive values of $\partial b / \partial z$. This is reflected in the strong positive value of the skewness, $S \approx 3$, a value which is significantly higher than the skewness observed for 


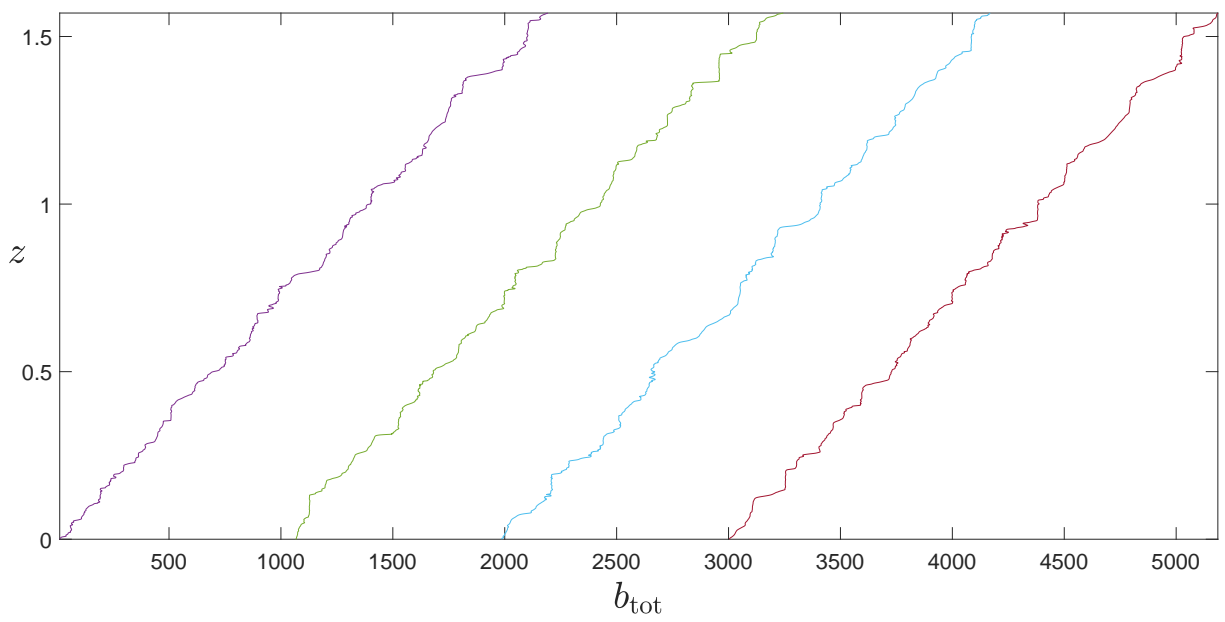

FiguRE 4 . Vertical profiles of $b_{\text {tot }}$ taken at four locations in the final snapshot of run R4. The profiles are at the following set of $x$-y grid points: [1 1], [6308 64], [3501 4096], [7000 6680]. From the second profile onward, the profiles are shifted along the $x$-axis for illustrative purpose.

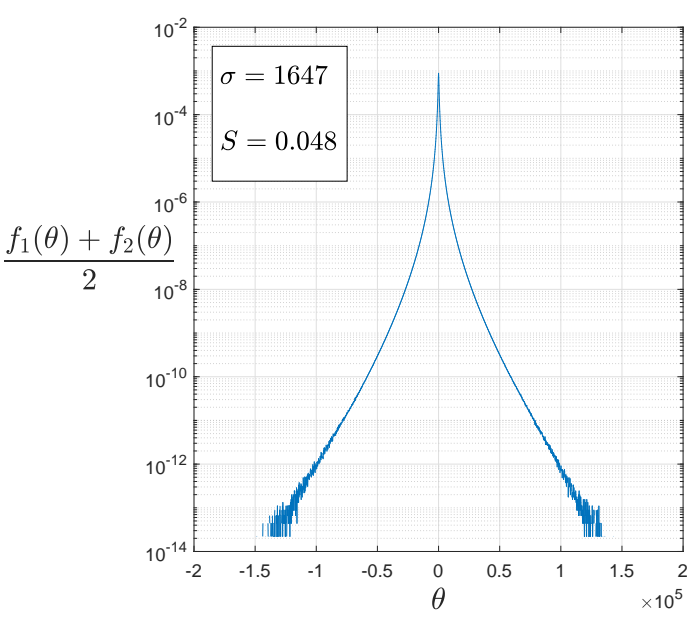

(a)

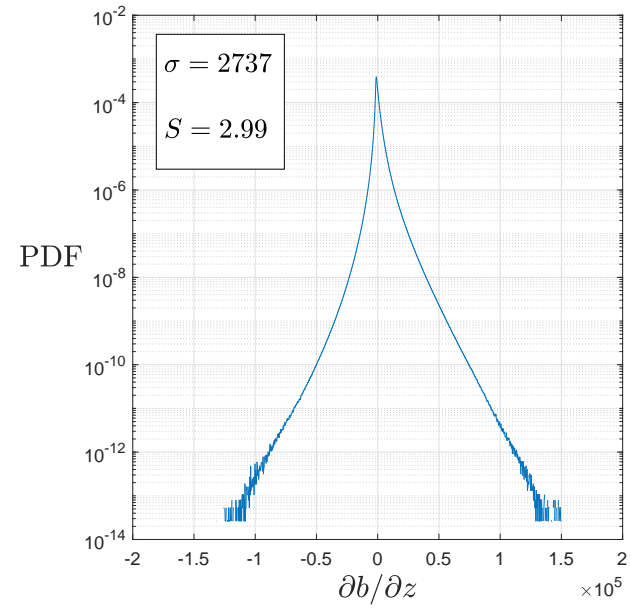

(b)

FiguRE 5. PDFs of horizontal and vertical buoyancy gradients for the final snapshot of run R4: (a) average of PDF of $\partial b / \partial x$ and of $\partial b / \partial y$, (b) PDF of $\partial b / \partial z$. In the subfigure (a), $f_{1}(\theta)$ is the $\mathrm{PDF}$ of $\partial b / \partial x, f_{2}(\theta)$ is the PDF of $\partial b / \partial y$ and $\theta$ is the sample-space variable.

passive scalar advection (Mydlarski \& Warhaft 1998) and also higher than the values of $S$ found previously for stratified turbulence (de Bruyn Kops 2015).

The PDFs of $\partial b / \partial z$ across the six DNS runs are shown in figure 6. Firstly, the shape of the PDF is noticeably asymmetric and positively skewed for all runs. Secondly, it seems that the skewness increases with increasing resolution, being small for run R1 and larger for run R4. This result is not immediately intuitive and appears to be in disagreement with our prediction of equation 2.18, according to which $S$ should be of order one and approximately constant. We will return to this question in the following section. Note that, as the resolution is increased, viscosity and diffusivity are decreased and the stratification is increased. This can be confirmed by inspection of the values of the non-dimensional parameters in table 1 . The Reynolds number increases in the 


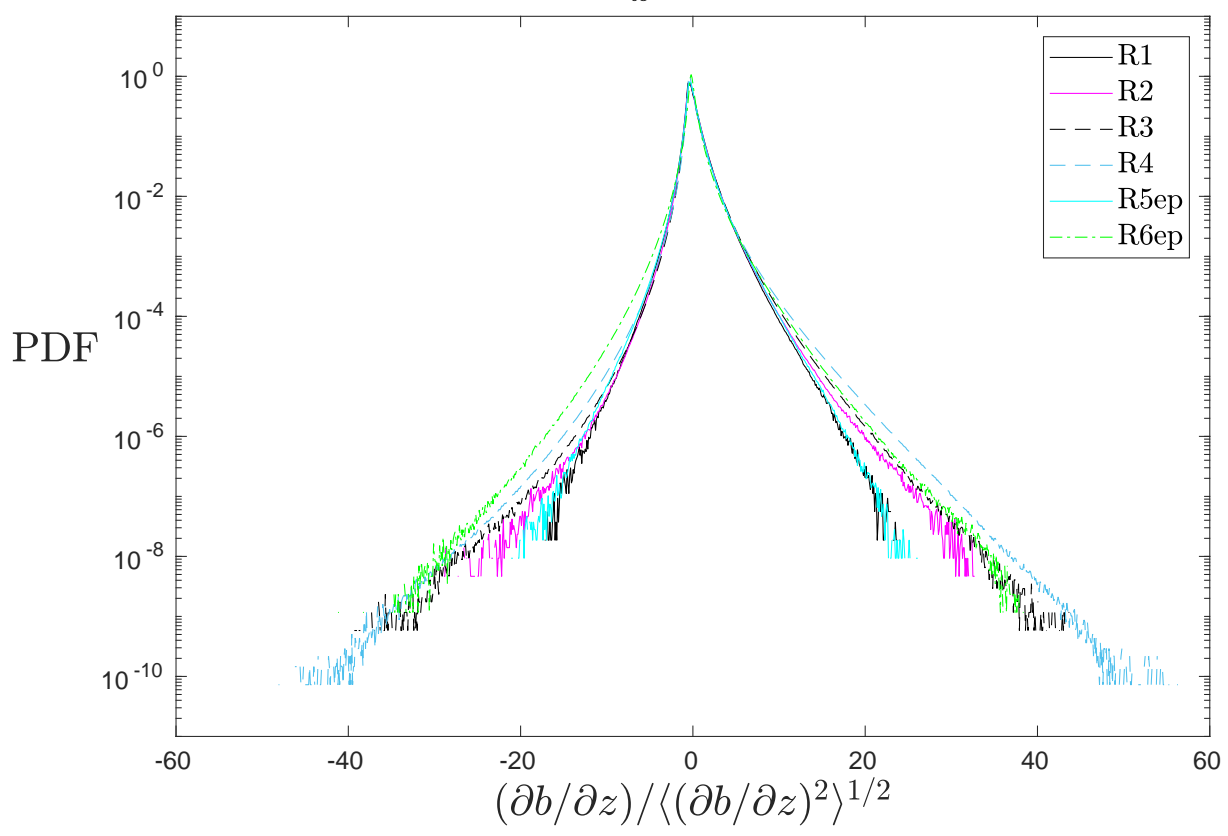

FIGURE 6. PDF of non-dimensional vertical buoyancy gradient for the final snapshot of each of the six simulations.

order $\operatorname{Re}(\mathrm{R} 1)<\operatorname{Re}(\mathrm{R} 5 \mathrm{ep})<\operatorname{Re}(\mathrm{R} 2)<\operatorname{Re}(\mathrm{R} 6 \mathrm{ep})<\operatorname{Re}(\mathrm{R} 3)<\operatorname{Re}(\mathrm{R} 4)$, following the resolution. On the other hand, for all runs except run R6ep the Froude number decreases continuously with resolution, $F_{h}(\mathrm{R} 1)>F r_{h}(\mathrm{R} 5 \mathrm{ep})>\operatorname{Fr}_{h}(\mathrm{R} 2)>F_{h}(\mathrm{R} 3)>F_{h}(\mathrm{R} 4)$. This is a result of the fact that for these five runs the buoyancy Reynolds number, $R e_{b}$, does not vary strongly. The above ordering then follows from the relation between these three parameters, $\operatorname{Re}_{b}=\operatorname{ReFr}_{h}^{2}$.

All the PDFs shown in figures 5 and 6 have been calculated from a single snapshot of each run taken at the end of the simulation. For runs R1, R2, R3 and R4 only this snapshot of the entire simulation was stored and the PDFs are available only at this time instant but for the final two runs, R5ep and R6ep, time-resolved PDFs are available. To obtain an idea of the spread of the statistics in these two runs, a plot of the mean PDF of $\partial b / \partial z$ throughout the statistically stationary period is given in figure 7 , including error bars corresponding to the standard deviation calculated for every sample value of $\partial b / \partial z$. The scatter of the PDFs is not large: for run R6ep the PDF at the final snapshot lies within the error bars and so does the PDF of run R5ep, except for very large positive values of buoyancy gradient.

\subsection{The scaling of the skewness in the DNS}

In this section we test the predictions of the scaling analysis of $\S 2$ and the expressions for the dimensional and non-dimensional skewness, $B_{z 3}$ and $S$. In order to do this, from the PDFs of $\partial b / \partial z$ shown in figure 6 , we have calculated values of $B_{z 2}, B_{z 3}$ and $S$ for each run. A positive test of equation 2.17 would give a linear relation between $B_{z 3}$ and $\epsilon^{3 / 2} N^{3} / \nu^{3 / 2}$. Figure 8 shows a plot of one quantity against the other. It is important to notice that all three physical quantities, $\epsilon, \nu$ and $N$, change significantly across the DNS runs, and so this represents a thorough test on the scaling of $B_{z 3}$. As can be seen from figure 8 , the agreement between $B_{z 3}$ from the DNS and its predicted scaling is remarkable. The scaling is seen to hold over five orders of magnitude in $B_{z 3}$. We 


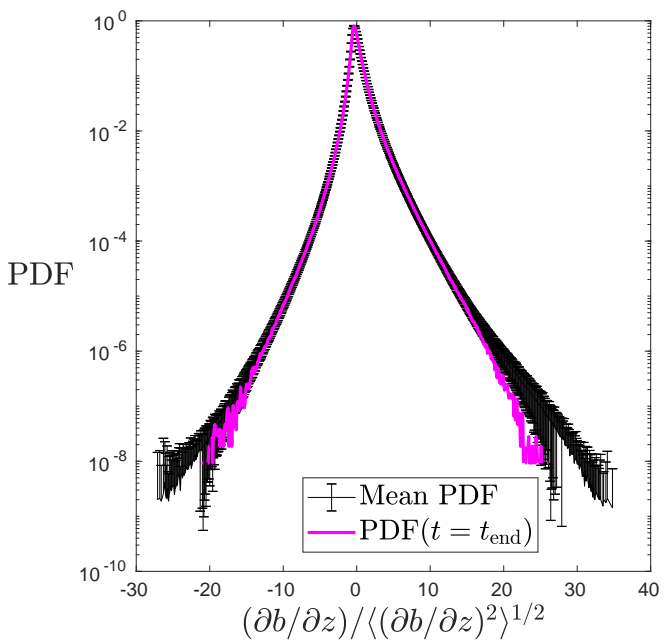

(a)

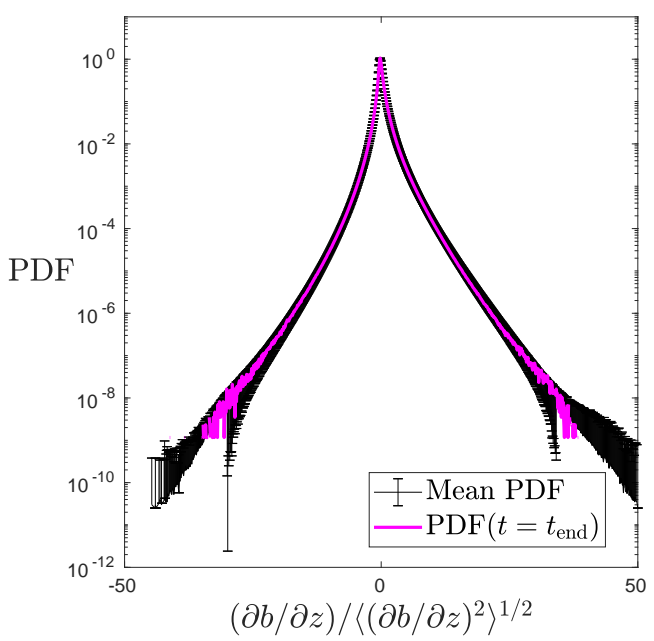

(b)

Figure 7. Comparison of mean PDF of $\partial b / \partial z$ of all the PDFs calculated during the statistically stationary period and PDF of the final snapshot: (a) run R5ep, (b) run R6ep. Error bars correspond to $\pm \sigma(\mathrm{PDF})$ calculated using all non-zero sample numbers in the $O(100)$ PDFs calculated over the statistically stationary period.

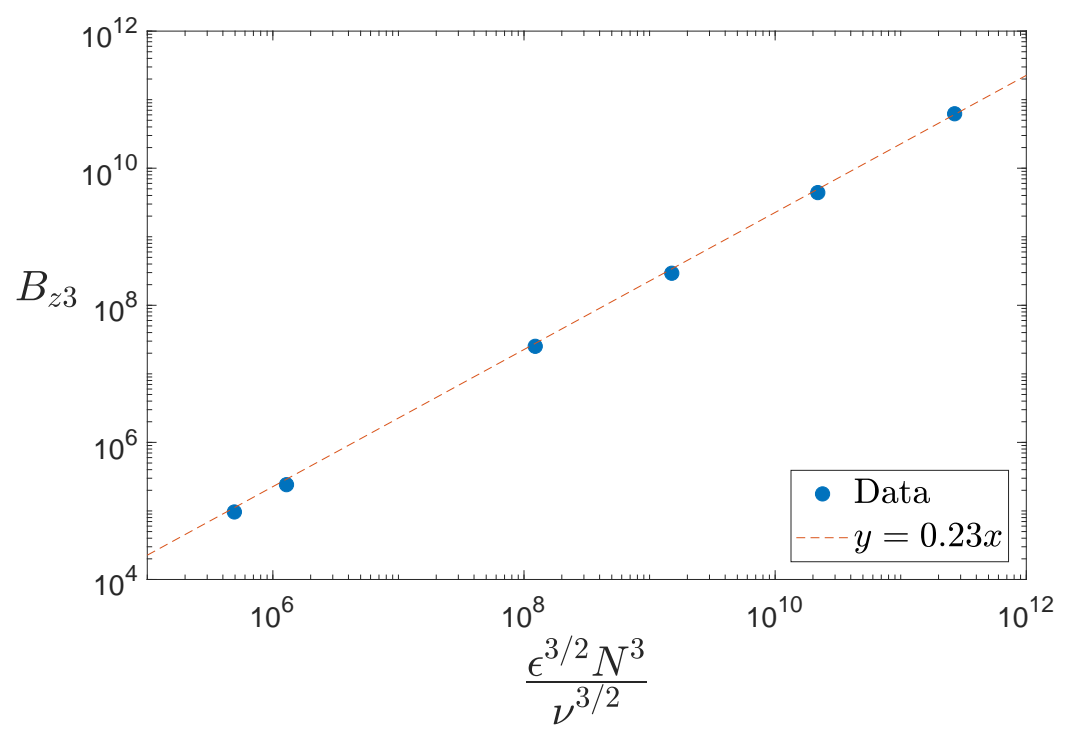

Figure 8. Dimensional skewness $B_{z 3}$ computed at the last time instant of the statistically stationary period for each DNS run as a function of $\epsilon^{3 / 2} N^{3} / \nu^{3 / 2}(\epsilon$ is not taken at this time but is a time average throughout the entire period of statistical stationarity). The data points, from left to right, correspond to runs R5ep, R6ep, R1, R2, R3, R4.

emphasize again that the thought experiment of the scaling analysis and the present DNS are significantly different, given the continuous presence of stratification (and of forcing) in the simulations. Despite this and the several assumptions made in deriving equation 2.17, the DNS are in good agreement with this result. The physical basis of equation 2.17 for the dimensional skewness is further confirmed by the fact that $B_{z 3}$ and $\epsilon^{3 / 2} N^{3} / \nu^{3 / 2}$ are of the same order of magnitude. 


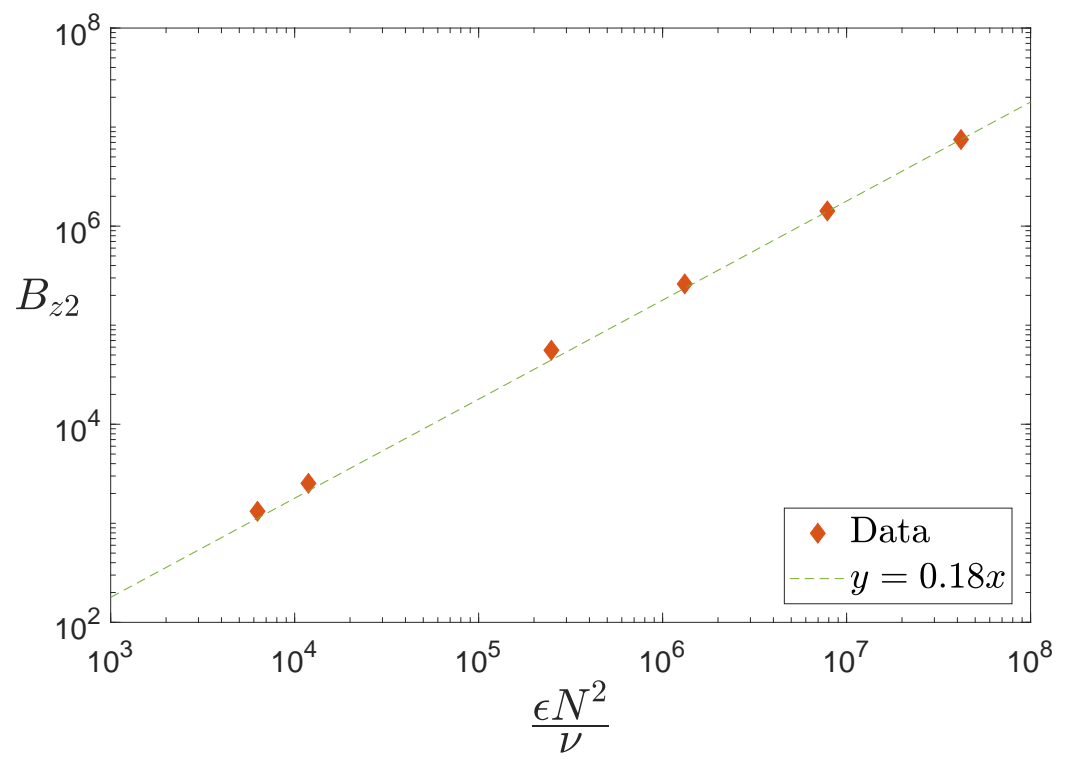

Figure 9. Variance of $\partial b / \partial z$ at the last time instant of each DNS run as a function of $\epsilon N^{2} / \nu$. The data points have the same correspondence as in figure 8 .

It is also worth verifying if the scaling for the variance of vertical buoyancy gradient, which we used to obtain the expressions for $B_{z 3}$ and $S$, is approximately met in the DNS. This scaling is $B_{z 2} \sim \epsilon N^{2} / \nu$. A plot of $B_{z 2}$ against its expected scaling is given in figure 9 . There is again a good agreement between values from the simulations and the prediction.

We now consider the behaviour of the skewness of vertical buoyancy gradient and test whether there is agreement between equation 2.18 and DNS results. In figure 10, the skewness is plotted as a function of the horizontal Froude number. It is clear that, as anticipated previously, the skewness varies somewhat across the six DNS runs and is not a constant as predicted by the scaling analysis. Since $S=B_{z 3} / B_{z 2}^{3 / 2}$, this result may be surprising but is probably due to the weak departures of $B_{z 2}$ and $B_{z 3}$ from their expected scalings (partially visible in figures 8-9). These small departures have a significant effect on $S$ because it is the fraction of two large numbers, which are of the same order of magnitude. The outcome is that there appears to be a systematic increase in $S$ with decreasing $\mathrm{Fr}_{h}$, which is not accounted for by the theory. It should also be mentioned that the dependence of $S$ on $F r_{h}$ is approximately given by $S \sim F r_{h}^{-0.41}$, and so is not a strong dependence. The variation of $S$ recast in terms of the Reynolds number (not shown) does not provide a clear dependence of $S$ on $R e$. This can be appreciated by the fact that runs R1 and R6ep have similar values of $S$ but significantly different values of $R e$. This is probably because $F r_{h}$ and not $R e$ is the parameter determining the variation of $S$. In terms of the magnitude of $S$, the scaling analysis is successful in predicting an $O(1)$ skewness, as observed in all the DNS runs.

\section{Discussion and conclusions}

The DNS results have confirmed some of the predictions of the scaling analysis based on the simple physical picture of the response of an isotropic scalar field to the imposition of a stable density gradient. The simulations also highlight a discrepancy with the simple 


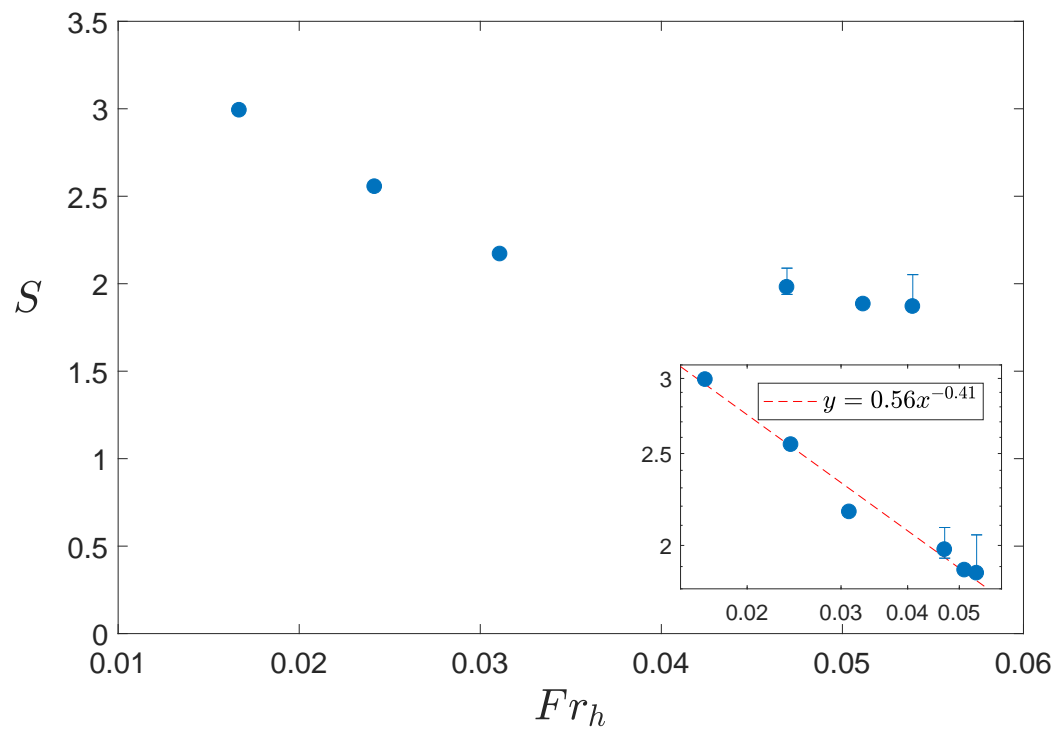

Figure 10. Skewness of $\partial b / \partial z$ as a function of $\mathrm{Fr}_{h}$. Log-log plot shown in inset including the best fit power law. Error bars are given for the data points corresponding to runs R5ep and R6ep and they show the minimum and maximum values of $S$ during steady state.

analysis in that the skewness of $\partial b / \partial z$ varies across the DNS runs, while maintaining a value of order one. The analysis has the merit of capturing the main trends in the variance and dimensional skewness of $\partial b / \partial z$. Altogether, these results confirm that there is a physical basis to the theoretical arguments of $\S 2$. It is therefore worth briefly reconsidering them in the light of the numerical simulations. In the DNS, stratification is present for the whole duration of the simulation and the initial condition for $b$ is not an isotropic and fully developed scalar field with a convective-inertial range, as in our thought experiment. In the simulations, the setup of forward transfers of buoyancy variance towards small scales and the growth of a positive skewness of $\partial b / \partial z$ rather take place simultaneously. The fact that the scaling for $B_{z 3}$ still holds, suggests these two processes are still related. In other words, a positive skewness still follows from a forward cascade of $b^{2}$ giving a negative $\left\langle\Delta u(\Delta b)^{2}\right\rangle$ at all scales. Hence, the continuous "rearrangement" of the gradients on a timescale $\tau \sim N^{-1}$ may be what leads to a positive skewness. This appears to be only a partial description of the mechanism leading to the buoyancy profiles of figure 4 with $S>0$. Nonetheless, if it is true it does show that non-linear transfers of $b^{2}$ to small scales are a necessary prerequisite, given which a positive skewness may grow on a linear timescale. So both linear and non-linear processes could be important in this problem.

The present DNS have shown that all cases become positively skewed, with relatively large values of $S$ in the range $S \approx 1.9-3$. These values are higher than those reported for the scalar gradient skewness (skewness $\approx 1$ ) in the passive scalar literature and are generally higher than, but consistent with the values, $S \approx 2-2.3$, for the two most strongly stratified DNS of de Bruyn Kops (2015). Hence the skewness of the buoyancy, an active scalar, is greater than the skewness of a passive scalar, both of which are in the presence of a mean gradient. The reason for this difference between the two cases could be that in the case of buoyancy there is an additional mechanism to the one we presented in $\S 2$ that can generate skewness. This mechanism is analogous to the centrifugal destabilization of anticyclones, which was used to explain the cycloneanticyclone asymmetry in rotating turbulence by Bartello et al. (1994). In stratified 
turbulence, the layers, which have a negative $\partial b / \partial z$, will become gravitationally unstable if $\partial b / \partial z<-N^{2}$, where $N^{2}$ is the background buoyancy gradient. Indeed, in this scenario $\partial b_{\text {tot }} / \partial z<0$ and the layer is gravitationally unstable, since it presents an overturning of the buoyancy field. This mechanism caps the negative buoyancy gradients of the layers to be at most $|\partial b / \partial z|=N^{2}$, which leads to an increase in the buoyancy gradient skewness. Since this mechanism is not present in passive scalar advection, it is likely the reason why the skewness is greater in stratified turbulence.

Considering the variation of $S$ expressed in terms of the horizontal Froude number, the DNS gave results close to a power law, $S \sim \mathrm{Fr}_{h}^{-0.42}$. A similar power of the Froude number, $\mathrm{Fr}_{h}^{-1 / 2}$, has a particular significance in stratified turbulence since $\mathrm{Fr}_{h}^{-1 / 2}=$ $\ell_{b} / \ell_{o}$. Here $\ell_{b}=u_{h} / N$ is the buoyancy lengthscale and $\ell_{o}=\sqrt{\epsilon / N^{3}}$ is the Ozmidov lengthscale. It is well known that $\ell_{b}$ is the vertical height of the layers in stratified turbulence (Billant \& Chomaz 2001), while $\ell_{o}$ is the smallest lengthscale in the turbulence that is strongly influenced by the stratification. Considering that, where present, layers and interfaces are expected to be set up because of the presence of the stratification, it could be reasonable to expect that the height of the interfaces is the smallest lengthscale influenced by $N$, i.e. $h_{\text {interf }} \sim \ell_{0}$. This suggestion has long been made in the stratified turbulence community but, to the best of the author's knowledge, it has not been shown rigorously in a simulation or experiment. If we accept that $h_{\text {interf }} \sim \ell_{o}$, together with $h_{\text {layer }} \sim \ell_{b}$, and assume a highly simplified cartoon in which layers and interfaces are piecewise linear profiles of buoyancy with a constant buoyancy gradient, we obtain:

$$
\left\{\begin{array}{l}
B_{z L} \equiv\left|\frac{\partial b}{\partial z}\right|_{\text {layer }} \sim \frac{\Delta b_{L}}{\ell_{b}} \\
B_{z I} \equiv\left|\frac{\partial b}{\partial z}\right|_{\text {interf }} \sim \frac{\Delta b_{I}}{\ell_{o}}
\end{array}\right.
$$

where $\Delta b_{L}$ and $\Delta b_{I}$ are the absolute values of the changes in buoyancy across a layer and an interface, respectively. However, referring back to the $b$-profile within the staircase profile in figure 1 , it is clear that $\Delta b_{L}=\Delta b_{I}$. This leaves us with $B_{z I} / B_{z L} \sim \ell_{b} / \ell_{o}=$ $\mathrm{Fr}_{h}^{-1 / 2}$. It is clear that an increase in the ratio of the buoyancy gradients in this cartoon, $B_{z I} / B_{z L}$, would make a layer-interface "step" sharper and increase the buoyancy gradient skewness, $S$. This provides a tentative rationalization of the observed dependency of $S$ on the Froude number. It is believed that this rationalization captures the long-time evolution of layers and interfaces, which depends on the detailed distribution of kinetic and potential energy across scales in stratified turbulence. As such, it cannot be captured by our theoretical considerations of $\S 2$, which are based on a rapid adjustment process.

In the light of the present results for the vertical buoyancy gradient skewness and of the above cartoon, we can revisit the physical mechanisms that create and sustain the layer-interface structure in stratified turbulence. A discussion on the dynamics necessarily requires consideration of the velocity field, which we have thus far left aside. A good starting point is the vertical energy spectrum $E_{h}\left(k_{v}\right)$, where $k_{v}$ is the vertical wavenumber. The vertical spectrum of the large horizontal scales, i.e. of the layers, was found recently to be $E_{\text {large }}\left(k_{v}\right) \sim N^{2} k_{v}^{-3}$ in simulations of stratified turbulence from $k_{v} \approx k_{b}=1 / \ell_{b}$ up to $k_{v} \approx k_{o}=1 / \ell_{o}$ (Maffioli 2017), as predicted theoretically by Billant \& Chomaz (2001). This means that the scale-by-scale gradient Richardson number is $R i_{g}\left(k_{v}\right)=N^{2} / S\left(k_{v}\right)^{2} \sim N^{2} /\left(k_{v}^{3} E_{\text {large }}\left(k_{v}\right)\right) \sim 1$, with the shear $S\left(k_{v}\right)$ calculated over vertical scales going from the buoyancy lengthscale $\ell_{b}$ to the Ozmidov lengthscale $\ell_{o}$. It is clear that $R i_{g}\left(k_{v}\right) \sim 1$ over this range implies that shear instability could take place over vertical scales from $\ell_{b}$ down to $\ell_{o}$. It is suggested that shear instability does take 
place and leads to overturns that mix the fluid locally and create a layer, either through the mixed region collapse described in $\S 1$ or through merging of the horizontal series of billows or overturns that shear instabilities create. Note that even if shear instability occurred at the lower end of this range of vertical scales, at $\ell_{o}$, it is expected that the ensuing mixed region would grow vertically up to $\ell_{b}$, and at this point the vertical scale cannot grow further (Billant \& Chomaz 2001). This dynamical picture is speculative but it has the merit of providing a possible explanation for the presence of layers of height $\ell_{b}$, in agreement with the above cartoon for the layer-interface structure. It builds upon a recurring suggestion (see Lilly 1983; Riley \& de Bruyn Kops 2003) that shear instabilities are important in stratified turbulence. More than a layer formation mechanism, it is believed that the above provides a sustaining mechanism of the layer-interface structure in which vertical portions of this structure go unstable, depleting the interface that was part of the unstable portion and forming a new layer of size $\ell_{b}$, which will be separated from the remaining structure by new interfaces of size $\ell_{o}$, and the process then repeats in a cyclic manner.

As a final point, we consider Warhaft's discussion of passive scalar advection in the presence of a mean scalar gradient in the limit $R e \rightarrow \infty$. Warhaft argues that strong interfaces in the total scalar concentration will remain in this limit, resulting in the small scales being anisotropic (due to the asymmetry along the direction of the mean scalar gradient) at all Reynolds numbers (Warhaft 2000). According to this work, this means that Kolmogorov's hypothesis of small-scale isotropy is contravened (Warhaft 2000). We consider this question in stratified turbulence. The specification of the limit of large Reynolds number is not enough, since in stratified turbulence there are two control parameters, the Froude number and the Reynolds number or the Froude number and the buoyancy Reynolds number, as we will use. The relevant limit for strongly stratified turbulence is $\mathrm{Fr}_{h} \rightarrow 0$ and $\mathrm{Re}_{b} \rightarrow \infty$, which corresponds to the limit of an infinitely large range of scales between $L$ and $\ell_{o}$ and of an infinitely large range of scales between $\ell_{o}$ and $\eta$. In terms of the distribution of $\partial b / \partial z$ across these scales, we expect that this will be maximum at the smallest scales, so somewhere close to the Kolmogorov lengthscale $\eta$. However, in the above cartoon of the layer-interface model, $\partial b / \partial z$ is concentrated in the interfaces and so is maximum at the Ozmidov lengthscale $\ell_{o}$. It could be that as $R e_{b} \rightarrow \infty$, the maximum buoyancy gradient moves from $\ell_{o}$ to $\eta$ as we effectively would have isotropic turbulence with a passive scalar and a mean scalar gradient below $\ell_{o}$, and a convective-inertial range for $\ell_{0} \gg r \gg \eta$. It is possible that in this scenario the skewness would start decreasing towards the value observed for passive scalar advection. This is an open question which we believe deserves further attention.

I am grateful to Y. Kimura for an insightful discussion on this subject. This manuscript has benefited from the comments and suggestions of anonymous referees. In particular, one anonymous referee is thanked for suggesting to work out the Prandtl number dependence of the skewness in the case of a passive scalar and for pointing me towards the relevant literature. This work was performed within the framework of the LABEX iMUST (ANR-10-LABX-0064) of Université de Lyon, within the program "Investissements d'Avenir" (ANR-11-IDEX-0007) operated by the French National Research Agency (ANR). I acknowledge PRACE for awarding us access to Fermi at CINECA, Italy, and to Marconi at CINECA, Italy. The PRACE project principal investigator is E. Lindborg, who I thank for many discussions on stratified turbulence. Additional computations were carried out in Sweden, at PDC, and I acknowledge SNIC for providing these resources. 


\section{REFERENCES}

Bartello, P., Métais, O. \& Lesieur, M. 1994 Coherent structures in rotating threedimensional turbulence. J. Fluid Mech. 273, 1-29.

Billant, P. \& Chomaz, J.-M. 2000 Experimental evidence for a new instability of a vertical columnar vortex pair in a strongly stratified fluid. J. Fluid Mech. 418, 167-188.

Billant, P. \& Chomaz, J.-M. 2001 Self-similarity of strongly stratified inviscid flows. Phys. Fluids 13 (6), 1645-1651.

Bos, W.J.T. 2014 On the anisotropy of the turbulent passive scalar in the presence of a mean scalar gradient. J. Fluid Mech. 744, 38-64.

Brethouwer, G., Billant, P., Lindborg, E. \& Chomaz, J.-M. 2007 Scaling analysis and simulation of strongly stratified turbulent flows. J. Fluid Mech. 585, 343-368.

Browand, F. K., Guyomar, D. \& Yoon, S.-C. 1987 The behavior of a turbulent front in a stratified fluid: experiments with an oscillating grid. J. Geophys. Res. 92 (C5), 5329-5341.

DE BRUyn Kops, S. M. 2015 Classical scaling and intermittency in strongly stratified boussinesq turbulence. J. Fluid Mech. 775, 436-463.

DAVIDSON, P. A. 2013 Turbulence in rotating, stratified and electrically conducting fluids. Cambridge University Press.

Desaubies, Y. \& GregG, M.C. 1981 Reversible and irreversible finestructure. J. Phys. Oceanog. 11, 541-556.

Deusebio, E., Boffetta, G., Lindborg, E. \& Musacchio, S. 2014 Dimensional transition in rotating turbulence. Phys. Rev. E 90 (023005).

Donzis, D.A., Sreenivasan, K.R. \& Yeung, P.K. 2005 Scalar dissipation rate and dissipative anomaly in isotropic turbulence. J. Fluid Mech. 532, 199-216.

Eswaran, V. \& Pope, S. B. 1988 An examination of forcing in direct numerical simulations of turbulence. Comput. Fluids 16 (3), 257-278.

Gan, L., Baqui, Y. B. \& Maffioli, A. 2016 An experimental investigation of forced steady rotating turbulence. Eur. J. Mech. B/Fluids 58, 59-69.

Gence, J.-N. \& Frick, C. 2001 Naissance des corrélations triples de vorticité dans une turbulence statistiquement homogène soumise à une rotation. C. R. Acad. Sci. Paris 329 (II b).

Gregg, M. C., D’Asaro, E. A., Riley, J. J. \& Kunze, E. 2018 Mixing efficiency in the ocean. Annu. Rev. Mar. Sci. 10 (1), 443-473.

Holford, J.M. \& Linden, P.F. 1999 Turbulent mixing in a stratified fluid. Dyn. Atmos. Oceans 30, 173-198.

Hopfinger, E. J., Browand, F. K. \& Gagne, Y. 1982 Turbulence and waves in a rotating tank. J. Fluid Mech. 125, 505-534.

Kimura, Y., Sullivan, P. \& Herring, J. 2016 Temperature front formation in stably stratified turbulence. International Symposium on Stratified Flows, vol. 1.

LiLLY, D.K. 1983 Stratified turbulence and the mesoscale variability of the atmosphere. $J$. Atmos. Sci. 40, 749-761.

Lindborg, E. 2006 The energy cascade in a strongly stratified fluid. J. Fluid Mech. 550, 207242.

Maffioli, A. 2017 Vertical spectra of stratified turbulence at large horizontal scales. Phys. Rev. Fluids 2, 104802.

Maffioli, A., Brethouwer, G. \& Lindborg, E. 2016 Mixing efficiency in stratified turbulence. J. Fluid Mech. 794, R3.

Maffioli, A., Davidson, P.A., Dalziel, S.B. \& Swaminathan, N. 2014 The evolution of a stratified turbulent cloud. J. Fluid Mech. 739, 229-253.

Mydlarski, L. \& WARhaft, Z. 1998 Passive scalar statistics in high-Peclet-number grid turbulence. J. Fluid Mech. 358, 135-175.

Park, Y.-G., Whitehead, J.A. \& Gnanadeskian, A. 1994 Turbulent mixing in stratified fluids: layer formation and energetics. J. Fluid Mech. 279, 279-311.

Phillips, O.M. 1972 Turbulence in a strongly stratified fluid is it unstable? 19 (1), 79-81.

Pinkel, R., Sherman, J., Smith, J. \& Anderson, S. 1991 Strain: observations of the vertical gradient of isopycnal vertical displacement. J. Phys. Oceanog. 21, 527-540. 
Posmentier, ERIC S 1977 The generation of salinity finestructure by vertical diffusion. J. Phys. Oceanog. 7 (2), 298-300.

Riley, J.J. \& De BRuYn Kops, S.M. 2003 Dynamics of turbulence strongly influenced by buoyancy. Phys. Fluids 15 (7), 2047-2059.

Riley, J. J. \& LELONG, M.-P. 2000 Fluid motions in the presence of strong stable stratification. Annu. Rev. Fluid Mech. 32, 613-657.

Rogallo, R. S. 1981 Numerical experiments in homogeneous turbulence. Tech. Rep. 81835. NASA Tech. Mem.

Schumacher, J. \& Sreenivasan, K.R. 2003 Geometric features of the mixing of passive scalars at high Schmidt numbers. Phys. Rev. Lett. 91 (17).

Staplehurst, P. J., Davidson, P. A. \& Dalziel, S. B. 2008 Structure formation in homogeneous freely decaying rotating turbulence. J. Fluid Mech. 598, 81-105.

TAYLOR, J.R. \& ZHOU, Q. 2017 A multi-parameter criterion for layer formation in a stratified shear flow using sorted buoyancy coordinates. J. Fluid Mech. 823, R5.

Thorpe, S.A. 2016 Layers and internal waves in uniformly stratified fluids stirred by vertical grids. J. Fluid Mech. 793, 380-413.

Warhaft, Z. 2000 Passive scalars in turbulent flows. Annu. Rev. Fluid Mech. 32, 203-240.

Watanabe, T. \& Gotoh, T. 2004 Statistics of a passive scalar in homogeneous turbulence. New J. Phys 6 (40).

Yaglom, A. M. 1949 On the local structure of the temperature field in a turbulent flow. Dokl. Akad. Nauk SSSR 69, 743.

Yeung, P. K., Xu, S. \& Sreenivasan, K. R. 2002 Schmidt number effects on turbulent transport with uniform mean scalar gradient. Phys. Fluids 14, 4178. 